Clinical Research

\title{
Significant changes in macrophage and CD8 T cell densities in primary prostate tumors 2 weeks after SBRT
}

\author{
Nathanael Kane ${ }^{1}$, Tahmineh Romero ${ }^{2}$, Silvia Diaz-Perez ${ }^{1}$, Matthew B. Rettig ${ }^{3}$, Michael L. Steinberg (D) ${ }^{1}$, Amar U. Kishan (iD ${ }^{1,3}$, \\ Dorthe Schaue $\mathbb{D}^{1}{ }^{1}$, Robert E. Reiter ${ }^{3}$, Beatrice S. Knudsen $\mathbb{I D}^{4}$ and Nicholas G. Nickols $\mathbb{D}^{1,3,5} \bowtie$
}

(c) The Author(s) 2022

\begin{abstract}
BACKGROUND: Radiotherapy impacts the local immune response to cancers. Prostate Stereotactic Body Radiotherapy (SBRT) is a highly focused method to deliver radiotherapy often used to treat prostate cancer. This is the first direct comparison of immune cells within prostate cancers before and after SBRT in patients.

METHODS: Prostate cancers before and 2 weeks after SBRT are interrogated by multiplex immune fluorescence targeting various T cells and macrophages markers and analyzed by cell and pixel density, as part of a clinical trial of SBRT neoadjuvant to radical prostatectomy.

RESULTS: Two weeks after SBRT, CD68, and CD163 macrophages are significantly increased while CD8 T cells are decreased. SBRT markedly alters the immune environment within prostate cancers.
\end{abstract}

Prostate Cancer and Prostatic Diseases (2023) 26:207-209; https://doi.org/10.1038/s41391-022-00498-6

Neoadjuvant trials of radiation prior to surgery offer the unique opportunity to study irradiated tissues in situ. We previously reported a Phase 1 trial of stereotactic body radiation therapy (SBRT), three fractions of $8 \mathrm{~Gy}$ delivered over 1 week, 2 weeks prior to radical prostatectomy (RP) for high-risk prostate cancer [1]. Pretreatment biopsies and surgical specimens from this trial were used to determine radiation-induced changes in T cell and macrophage subsets by multiplex immune-fluorescence (mIF).

The 6 out of 11 cases were chosen due to their availability of biopsy and RP cores for use. Biopsy cores were selected for analysis based on tumor content greater than $70 \%$. For each case, the tissue block with the dominant tumor nodule in the radical prostatectomy was identified by hematoxylin and eosin staining prior to $\mathrm{mIF}$. Parallel slides were stained with antibodies against T cells [CD3 (clone 2Gv6 rabbit mAb, Ventana cat. 790-4341), CD4 (clone SP35 rabbit mAb, Ventana cat 790-4423), CD8 (clone SP57 rabbit mAb, Ventana cat. 790-4460), FoxP3 (clone SP97 rabbit $\mathrm{mAb}$, Spring Bioscience cat. M3970)] or macrophages [CD68 (clone KP-1 mouse mAb, Ventana cat. 790-2931), CD163 (clone MRQ26 mouse mAb, Ventana cat. 760-4437), CD11b (clone EPR1344 rabbit mAb, Abcam cat. Ab133357)]. CD3 and CK8/18 (clone 5D3 mouse $\mathrm{mAb}$, Abcam cat. Ab17139) antibodies were included in both panels. FFPE tissue sections were stained on the Ventana Discovery Ultra autostainer, and analyzed using Tissuegnostics Strataquest software (v. 6.0.1.211). A representative image is shown in Fig. 1. RP regions went through additional manual quality control with the guidance of a pathologist to exclude normal glands that were intermixed with tumor glands. Image analysis included optimization of IF thresholding and nuclear segmentation. A CK 8/18 defined mask in tumor regions was expanded by 20 micron to generate a ring of $\sim 1-2$ full cell lengths around tumor glands and to enumerate $\mathrm{T}$ cell and macrophage pixel numbers, as described previously [2].

We included each variable ( $T$ cell or macrophage densities or percentages of positive tumor region in pixels) as the dependent variable of a generalized linear mixed model (GLMM) for repeated measures in which RP vs Biopsy (RP $y / n$ ) was the independent covariate under a compound symmetry covariance structure matrix. All tests were two sided with $p<0.05$ considered statistically significant. We used R (R version 3.6.1 (2019-07-05) and LME4 package and SAS (9.4, SAS Institute Inc., Cary, NC, USA) to analyze the data.

CD8 T cell $(C D 3+C D 8+)$ densities within tumor regions after SBRT were decreased 2.22 -fold $(p=0.001)$ relative to densities before SBRT, while CD4 T cell $(C D 3+C D 4+)$ densities, and Treg cell (CD4 + FOXp3) densities remained largely unchanged. The most striking change was the increase in CD11b + myeloid cells, particularly the CD68 + macrophages, and CD163+ macrophage subsets that reached 1.85 -fold, 3.43-fold, and 5.61-fold change in densities after SBRT, respectively (Fig. 1).

Altogether, the immune environment within prostate tumors is significantly altered 2 weeks after SBRT. This is the first report to quantify infiltrating immune cells in situ within prostate tumors to determine local effects of SBRT in patients. This is in line with our previous flow cytometric investigation showing an equivalent shift in infiltrating immune cells post SBRT towards myeloid lineage predominance although tumor and non-tumor regions

\footnotetext{
'Department of Radiation Oncology, David Geffen School of Medicine at UCLA, Los Angeles, CA, USA. ${ }^{2}$ Statistic Core, David Geffen School of Medicine at UCLA, Los Angeles, CA, USA. ${ }^{3}$ Department of Urology, David Geffen School of Medicine at UCLA, Los Angeles, CA, USA. ${ }^{4}$ Department of Pathology, University of Utah, Salt Lake City, UT, USA. ${ }^{5}$ Radiation Oncology Service, VA Greater Los Angeles Healthcare System, Los Angeles, CA, USA. ${ }^{\varpi}$ email: nnickols@mednet.ucla.edu
}

Received: 14 October 2021 Revised: 5 January 2022 Accepted: 12 January 2022

Published online: 20 January 2022 
A)
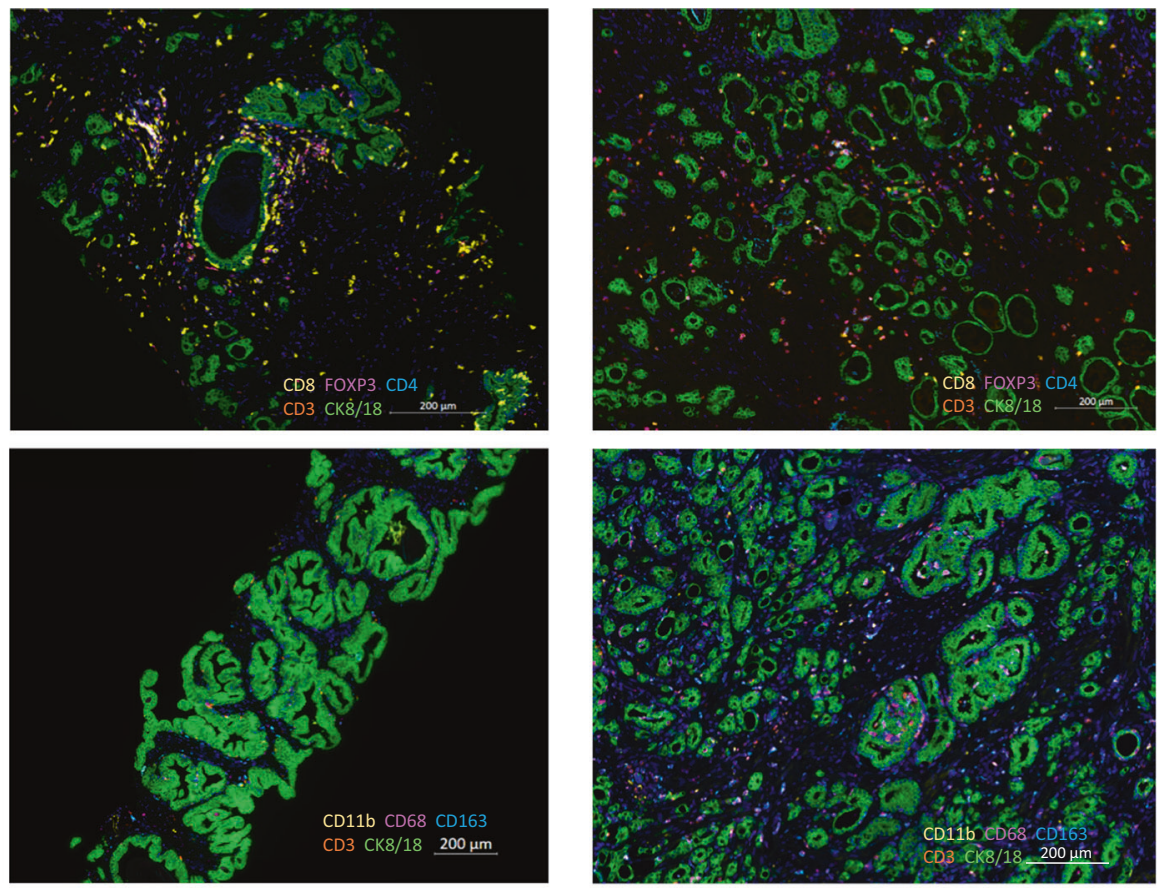

B)
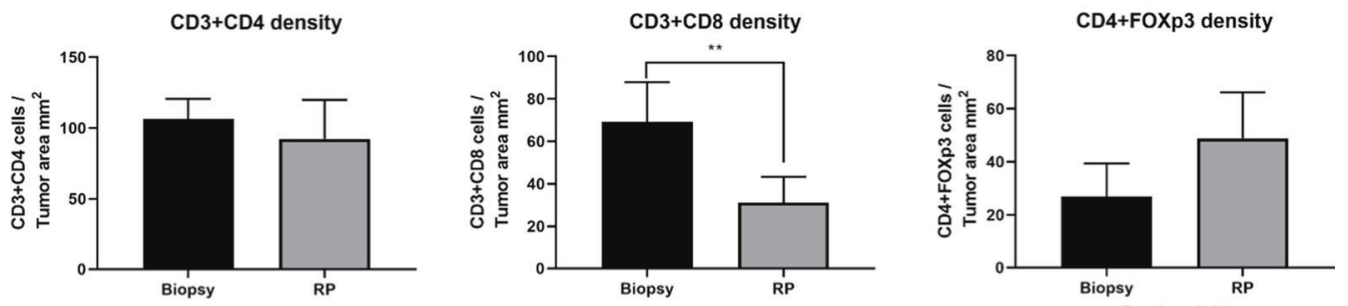

P-value: 0.718
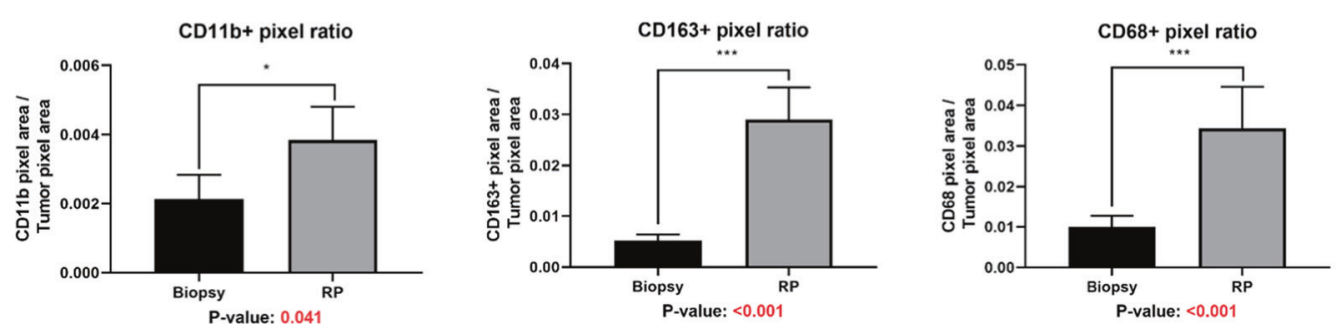

C)

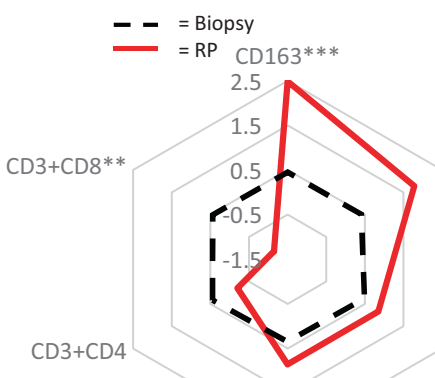

D)

\begin{tabular}{lcccc} 
cStage & Gleason Grade & Cores positive & iPSA & pStage \\
\hline 1 cT2bNoMo & $4+4=8 /$ GG 4 & 11 of 13 & 7.9 & pT3aNoMo \\
2 cT1cNoMo & $4+5=9 /$ GG5 & 11 of 12 & 11.1 & pT3aNoMo \\
3 cT2aNoMo & $4+5=9 /$ GG5 & 8 of 12 & 5.9 & pT3aNoMo \\
4 cT1cN1Mo & $4+3=7 /$ GG3 & 3 of 12 & 24.1 & pT3bNIMo \\
5 cT3aN1Mo & $4+4=8 /$ GG4 & 7 of 14 & 4.5 & pT3aNIMo \\
6 cT2cN1Mo & $4+3=7 /$ GG3 & 12 of 14 & 18.3 & pT3bNIMo
\end{tabular}

CD4+FOXp3

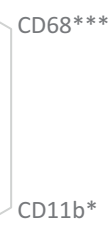

Fig. 1 Changes in T cell and macrophage densities before and after prostate SBRT. A Representative multiplex IF images of a panel of T cell markers in pre-SBRT biopsies (top left) and post- SBRT RP specimens (top right) along with a panel of macrophage cell markers in biopsies (bottom left) and RP (bottom right). Images were taken using ZEN 3.1 imaging software. Sequential slides were cut at $4 \mu \mathrm{m}$ prior to staining. The T cell panel included immune-fluorescent (IF) antibody-fluorophore pairs CD4-DCC, CD8-Rhod6G, CD3-Red610, FoxP3-Cy5, cytokeratin 8/18FAM and DAPI. The macrophage panel included CD163-DCC, CD11b-Rhod6G, CD3-Red610, CD68-Cy5, cytokeratin 8/18-FAM and DAPI. B Bar graphs of T cells (top row) and macrophage cells (bottom row) within tumor regions. Means are shown with SEM error bars. T cells are counts of cells per unit area. Macrophages are positive pixels per unit area. C Polar graph using mean log2-fold differences between RP samples and biopsy samples $\log 2$ (mean of RP/mean of biopsy). ${ }^{*} p<0.05,{ }^{* *} p<0.01,{ }^{* * *} p<0.001$. D Clinical and pathologic features of cases analyzed. 
could not be distinguished at that time [3]. By spatially resolving tumor and non-tumor glands in situ, we demonstrate significant increases in macrophage densities and moderate reductions in CD8 T cell densities 2 weeks post SBRT. In a syngeneic murine model, we previously reported increases in myeloid cells 2 days after high dose per fraction radiation, followed by a transient increase of functionally active CD8 lymphocytes [4]. The changes we report here in macrophage and CD8 T cell densities 2 weeks post SBRT in patients may also be time dependent, but our analysis was limited to a single time point given the design of the clinical trial. Similar digital spatial profiling of prostate tissue before and after 2 weeks of $10 \mathrm{~Gy}$ delivered by HDR brachytherapy also indicated such an increase in macrophage subpopulations in the post-radiation phase [5]. The fact that this was accompanied by a strong tumor inflammatory signature points at an integrated, myeloid-centric tissue response that radiation damage is known to initiate. To reveal underlying cytokines and mechanisms that are responsible for observed changes in the immune cell infiltration, transcriptomic analyses on prostate cancer tissue post SBRT are warranted. Ultimately, the questions will be whether or not these myeloid subsets are hindering the anti-tumor immune response and if so, how they can be targeted.

\section{REFERENCES}

1. Parikh NR, Kishan AU, Kane N, Diaz-Perez S, Ganapathy E, Nazarian R, et al. Phase 1 trial of stereotactic body radiation therapy neoadjuvant to radical prostatectomy for patients with high-risk prostate cancer. Int J Radiat Oncol Biol Phys. 2020;108:930-5. https://doi.org/10.1016/j.ijrobp.2020.06.010

2. Saylor J, Ma Z, Goodridge HS, Huang F, Cress AE, Pandol SJ, et al. Spatial mapping of myeloid cells and macrophages by multiplexed tissue staining. Front Immunol. 2018;9:2925. https://doi.org/10.3389/fimmu.2018.02925

3. Nickols NG, Ganapathy E, Nguyen C, Kane N, Lin L, Diaz-Perez S, et al. The intraprostatic immune environment after stereotactic body radiotherapy is dominated by myeloid cells. Prostate Cancer Prostatic Dis. 2021;24:135-9. https://doi.org/ 10.1038/s41391-020-0249-8

4. Lin L, Kane N, Kobayashi N, Kono EA, Yamashiro JM, Nickols NG, et al. High-dose per fraction radiotherapy induces both antitumor immunity and immunosuppressive responses in prostate tumors. Clin Cancer Res. 2021;27:1505-15. https:// doi.org/10.1158/1078-0432.CCR-20-2293
5. Keam SP, Halse H, Nguyen T, Wang M, Van Kooten Losio N, Mitchell C, et al. High dose-rate brachytherapy of localized prostate cancer converts tumors from cold to hot. J Immunother Cancer. 2020;8. https://doi.org/10.1136/jitc-2020-000792.

\section{AUTHOR CONTRIBUTIONS}

NK, SDP, AUK, DS, RER, BSK, NGN designed the experiments. NK, SDP conducted the experiments. NK, SDP, TR, MBR, MLS, AUK, DS, RER, BSK, NGN analyzed data. All authors contributed to writing or critical review of the paper. NN, RER, BSK provided funding, administration, and/or resources.

\section{COMPETING INTERESTS}

NN reports research support from Lantheus, Bayer, Janssen, and consulting fees from Oncolinea, outside of the scope of work.

\section{ADDITIONAL INFORMATION}

Correspondence and requests for materials should be addressed to Nicholas G. Nickols.

Reprints and permission information is available at http://www.nature.com/ reprints

Publisher's note Springer Nature remains neutral with regard to jurisdictional claims in published maps and institutional affiliations.

\begin{abstract}
Open Access This article is licensed under a Creative Commons Attribution 4.0 International License, which permits use, sharing, adaptation, distribution and reproduction in any medium or format, as long as you give appropriate credit to the original author(s) and the source, provide a link to the Creative Commons license, and indicate if changes were made. The images or other third party material in this article are included in the article's Creative Commons license, unless indicated otherwise in a credit line to the material. If material is not included in the article's Creative Commons license and your intended use is not permitted by statutory regulation or exceeds the permitted use, you will need to obtain permission directly from the copyright holder. To view a copy of this license, visit http://creativecommons. org/licenses/by/4.0/.
\end{abstract}

(c) The Author(s) 2022 